Groups Geom. Dyn. 9 (2015), 133-148

DOI $10.4171 / \mathrm{GGD} / 308$
Groups, Geometry, and Dynamics

(C) European Mathematical Society

\title{
Superrigidity in infinite dimension and finite rank via harmonic maps
}

\author{
Bruno Duchesne ${ }^{1}$
}

\begin{abstract}
We consider infinite dimensional Riemannian manifolds of nonpositive curvature and finite telescopic dimension. We prove geometric superrigidity for actions of cocompact lattices in semisimple Lie groups of higher rank on theses spaces.
\end{abstract}

Mathematics Subject Classification (2010). 53C35, 53C43, 22E40.

Keywords. Infinite dimensional symmetric spaces, harmonic maps, superrigidity.

\section{Introduction}

1.1. Geometric superrigity. In the nineteen seventies, Margulis proved his famous superrigidity theorem to show that irreducible lattices in higher rank semisimple Lie groups and algebraic groups are arithmetic.

Theorem 1.1 ([24]). Let $G, H$ be semisimple algebraic groups over local fields without center nor compact factors. Assume that the real rank of $G$ is at least 2 and let $\Gamma$ be an irreducible lattice of $G$.

Any homomorphism $\Gamma \rightarrow H$ with unbounded and Zariski dense image extends to a homomorphism $G \rightarrow H$.

Using the dictionary between semisimple algebraic groups over local fields and symmetric spaces of noncompact type (in the Archimedean case) and Euclidean buildings (in the non-Archimedean case), Theorem 1.1 can be interpreted in a geometric way. This is the subject of the so-called geometric superrigidity, see [29] for a survey in French or the older [10]. Using this geometric interpretation, Corlette [5] (in the Archimedean case) and later Gromov and Schoen [12] extended

\footnotetext{
${ }^{1}$ The author was supported by a postdoctoral fellowship of the Swiss National Science Foundation.
} 
Margulis superridigity theorem in the case where $G$ is a simple Lie group of rank 1 that is the isometry group of a quaternionic hyperbolic space or the isometry group of the Cayley hyperbolic plane. The main tool in these two former results are harmonic maps. Some time later, harmonic maps allowed Mok, Siu and Yeung to give a very general statement [27] of geometric superrigidity in the Archimedean case. A similar result was also obtained by Jost and Yau in [15].

The framework of geometric superrigidity was extended $[25,9]$ to nonpositively curved metric spaces, which may be not locally compact, in the particular higher rank case where $\Gamma$ is a lattice in a product.

In [11, 6.A], Gromov invited geometers to study some "cute and sexy" infinite dimensional symmetric spaces of nonpositive curvature and finite rank. These spaces are

$$
X_{p}(\mathbb{R})=\mathrm{O}(p, \infty) /(\mathrm{O}(p) \times \mathrm{O}(\infty)) .
$$

They have analogs, denoted by $X_{p}(\mathbb{K})$, over the field $\mathbb{K}$ of complex or quaternionic numbers. Their geometry was studied in [8]. Gromov also conjectured that actions of lattices in semisimple Lie groups on some $X_{p}(\mathbb{R})$ should be subject to geometric superrigidity.

In this article, a Riemannian manifold will be a connected smooth manifold modeled on a separable Hilbert space and endowed with a smooth Riemannian metric. In particular, such a manifold may have infinite dimension. See [17] or [22] for an accurate definition.

The main result of this paper is the following theorem.

Theorem 1.2. Let $\Gamma$ be an irreducible torsion free uniform lattice in a connected higher rank semisimple Lie group with finite center and no compact factor G. Let $N$ be a simply connected complete Riemannian manifold of nonpositive sectional curvature and finite telescopic dimension.

If $\Gamma$ acts by isometries on $N$ without fixed point in $N \cup \partial N$ then there exists a $\Gamma$-equivariant isometric totally geodesic embedding of a product of irreducible factors of the symmetric space of $G$ in $N$.

In the unpublished paper [20], Korevaar and Schoen introduced the notion of FR-spaces (Finite Rank spaces). Later, Caprace and Lytchak introduced the notion of spaces of finite telescopic dimension in [2], without knowing [20]. The two notions are the same for complete $\operatorname{CAT}(0)$ spaces and can be defined by an 
inequality at large scale inspired by Jung inequality. For any bounded subset $Y \subset$ $\mathbb{R}^{n}$, Jung proved that

$$
\operatorname{rad}(Y) \leq \sqrt{\frac{n}{2(n+1)}} \operatorname{diam}(Y),
$$

see [23] and references therein. So, a complete CAT(0) space $X$ has telescopic dimension less than $n$ if for any $\delta>0$ there exists $D>0$ such that for any bounded subset $Y \subseteq X$ of diameter larger than $D$, we have

$$
\operatorname{rad}(Y) \leq\left(\delta+\sqrt{\frac{n}{2(n+1)}}\right) \operatorname{diam}(Y) .
$$

Theorem 1.2 applies to the particular case where $N$ is a symmetric space of noncompact type and finite rank (see [7] for the meaning of noncompact type in infinite dimension). The fact that symmetric spaces of noncompact type and finite rank have finite telescopic dimension was expected in [20] and proved in Corollary 1.8 of [7]. Actually, it is proved that a symmetric space of noncompact type is a finite product of irreducible symmetric spaces of noncompact type and that irreducible factors of infinite dimension are some $X_{p}(\mathbb{K})$.

This theorem implies that there is no geometrically Zariski-dense (see $[26,5 . B])$ action of a uniform lattice as in Theorem 1.2, on a symmetric space of noncompact type, infinite dimension and finite rank. In rank 1, it was shown that the isometry group of the real hyperbolic space $\mathbf{H}^{n}$ has geometrically Zariskidense actions on the infinite dimensional hyperbolic $\mathbf{H}^{\infty}$, see [26].

The strategy to prove Theorem 1.2 goes as follows. First, there is a harmonic map when the target is considered to be only a CAT(0) space. When the target is moreover a Riemannian manifold, the unique harmonic map is smooth. The fact that the harmonic map is totally geodesic finishes the proof.

Remark 1.3. Theorem 1.2 extends to the case where $G$ is the connected component of the isometry group of a quaternionic hyperbolic space or of the Cayley hyperbolic plane and $N$ has nonpositive complexified sectional curvature. This last condition is satisfied when $N$ is a symmetric space of noncompact type.

1.2. A flat torus theorem for parabolic isometries. In the last section, we include an extension for parabolic isometries of the well-known flat torus theorem [1, Chapter II.7]. This extension allows us to obtain a rigidity statement easily. 
Let $\gamma$ be an isometry of a CAT( 0$)$ space $X$. The translation length of $\gamma$ is the number $|\gamma|=\inf _{x \in X} d(\gamma x, x) ; \gamma$ is said to be ballistic if $|\gamma|>0$ and neutral otherwise. Since the infimum in the definition of $|\gamma|$ may or may not be achieved, it is usual to distinguish between semisimple isometries, for which the infimum is a minimum, and parabolic isometries, for which the infimum is not a minimum. Let $\varphi: G \rightarrow \operatorname{Isom}(X)$ be a homomorphism. We say that $G$ acts by ballistic isometries on $X$ if $\varphi(g)$ is a ballistic isometry for any $g \neq e$.

A CAT(0) space $X$ is said to be $\pi$-visible if any points $\xi, \eta \in \partial X$ that satisfy $\angle(\xi, \eta)=\pi$ are extremities of a geodesic line. For example, Hilbert spaces, Euclidean buildings and symmetric spaces of noncompact type are $\pi$-visible. Let $H$ be a subset of $\operatorname{Isom}(X)$, we denote by $z_{\operatorname{Isom}(X)}(H)$ the centralizer of $H$, that is the set of elements in Isom $(X)$ that commute with all elements in $H$.

Theorem 1.4. Let $X$ be a complete $\pi$-visible $\mathrm{CAT}(0)$ space and let $A$ be an abelian free group of rank $n$ acting by ballistic isometries on $X$.

Then there exists a A-invariant closed convex subspace $Y \subseteq X$. The space $Y$ decomposes as $Z \times \mathbb{R}^{n}$, $\mathcal{Z}_{\operatorname{Isom}(X)}(A)$ preserves this decomposition and the action $Z_{\operatorname{Isom}(X)}(A) \curvearrowright Y$ is diagonal.

Moreover, the action $A \curvearrowright \mathbb{R}^{n}$ is given by a lattice of $\mathbb{R}^{n}$ acting by translations and for any $a \in A$, the action of $a$ on $Z$ is neutral.

Corollary 1.5. Let $\Gamma$ be a lattice in a semisimple Lie group of real rank $r$. If $\Gamma$ acts by ballistic isometries on a symmetric space of nonpositive curvature $X$ then $r \leq \operatorname{rank}(X)$.

Acknowledgments. The author thanks Pierre Pansu for suggesting this approach to superrigidity in infinite dimension and thanks Pierre Py for pleasant and useful discussions about regularity of harmonic maps in infinite dimension.

\section{Harmonic maps}

In this section, we recall the standard notions of totally geodesic maps and harmonic maps between Riemannian manifolds (maybe of infinite dimension). We refer to [28], among others, for an introduction to these notions in finite dimension.

Let $(M, g)$ be a smooth Riemannian manifold with Levi-Civita connection $\nabla$. Let $u$ be a chart from an open subset $U \subset M$ to an open subset $V$ of a Hilbert space $\mathcal{H}$. The restriction to $U$ of any vector field $X \in \Gamma(T M)$ can be thought as 
a smooth map $V \rightarrow \mathcal{H}$ and thus we can consider the differential $D X$ of $X$ as a linear map from $\mathcal{H}$ to itself. The Christoffel symbol of $\nabla, \Gamma(u)$ with respect to $u$, is defined by the relation

$$
\nabla_{Y} X=D_{Y} X+\Gamma(u)(X, Y),
$$

see [17, 1.5]. Let $f$ be a smooth map between Riemannian manifolds $(M, g)$ and $(N, h)$ with Levi-Civita connections ${ }^{M} \nabla$ and ${ }^{N} \nabla$. The vector bundle $f^{-1} T N$, which is the vector bundle over $M$ with fibers $T_{f(x)} N$ for $x \in N$, is endowed with the connection induced from ${ }^{N} \nabla$, which we denote also by ${ }^{N} \nabla$. In charts $(u, U),(v, V)$ of $M$ such that $f(U) \subset V$, this connection is given by the formula

$$
{ }^{N} \nabla_{X} Y=D_{d f X} Y+\Gamma(v)(d f X, Y)
$$

for $X \in \Gamma(T M)$ and $Y \in \Gamma\left(f^{-1} T N\right)$. The vector bundle $T M^{*}$ is also endowed with a connection ${ }^{M} \nabla^{*}$ induced from ${ }^{M} \nabla$. For $\omega \in \Gamma\left(T M^{*}\right)$ and $X, Y \in \Gamma(T M)$,

$$
{ }^{M} \nabla_{X}^{*} \omega(Y)=X \cdot \omega(Y)-\omega\left(\nabla_{X} Y\right) .
$$

The vector bundle $f^{-1} T N \otimes T M$ is endowed with the connection

$$
\nabla: \Gamma\left(T M^{*} \otimes f^{-1} T N\right) \longrightarrow \Gamma\left(T M^{*} \otimes T M^{*} \otimes f^{-1} T N\right)
$$

induced by ${ }^{M} \nabla^{*}$ and ${ }^{N} \nabla$. This connection is defined by the formula

$$
\nabla_{X}(\omega \otimes V)={ }^{M} \nabla_{X}^{*} \omega \otimes V+\omega \otimes \otimes^{N} \nabla_{X} V
$$

for $X \in \Gamma(T M), \omega \in \Gamma\left(T M^{*}\right)$ and $V \in \Gamma\left(f^{-1} T N\right)$.

The differential $d f$ of a smooth map $f$ is a section of $T M^{*} \otimes f^{-1} T N$ and $f$ is called totally geodesic if $\nabla d f=0$. One can think of this property in two equivalent ways. A map $f$ is totally geodesic if and only if it preserves the connections, that is ${ }^{N} \nabla_{X} d f Y=d f\left({ }^{M} \nabla_{X} Y\right)$ for $X, Y \in \Gamma(T M)$. And $f$ is totally geodesic if and only if it maps geodesics to geodesics.

When $M$ is finite dimensional, there is a more general notion. Let $\tau(f)$ be the trace of $\nabla d f$. It is a section of the vector bundle $T M^{*} \otimes T M^{*} \otimes f^{-1} T N$ defined by

$$
\tau(f)=\sum_{i} \nabla d f\left(e_{i}, e_{i}\right)
$$

for any orthonormal base $\left(e_{i}\right)$ of $T_{x} M$. The map $f$ is harmonic if $\tau(f)=0$. Harmonic maps are also solutions of a variational problem. Let $\|$ || be the norm 
associated to the Riemannian metric $g \otimes h$ on $T M^{*} \otimes f^{-1} T N$. Actually for $x \in M$, $\left\|d_{x} f\right\|$ is the Hilbert-Schmidt norm of the linear map $d_{x} f: T_{x} N \rightarrow T_{f(x)} N$. This norm is well defined because $T_{x} M$ is finite dimensional. If $M$ is complete and has finite Riemannian volume, the energy of $f$ is

$$
E(f)=\int_{M}\|d f\|^{2} .
$$

Harmonic maps are exactly critical points of the energy. There exists an equivariant version of this variational problem. Let $\Gamma$ be the fundamental group of a compact Riemannian manifold $M$ acting by isometries on $N$ and let $f$ be a $\Gamma$-equivariant map $f: \tilde{M} \rightarrow N$ where $\tilde{M}$ is the universal covering of $M *$. Since $\|d f\|^{2}$ is $\Gamma$-invariant, one can define the energy of $f$ by $E(f)=\int_{M}\|d f\|^{2}$. In the case where $N$ is finite dimensional and non positively curved, the existence of equivariant harmonic maps was obtained in $[4,21]$.

\section{Harmonic maps for metric spaces targets}

In [18, 19], Korevaar and Schoen developed a theory of harmonic maps with metric spaces targets (Jost developed also a similar theory, see [13], [16] or [14, 8.2]). We recall the definitions (not in full generality but in a framework convenient to our purpose) and refer to the original papers for details.

Let $(\Omega, \mu)$ be a standard measure space with finite measure and let $(X, d)$ be a complete separable metric space with base point $x_{0}$. The space $L^{p}(\Omega, X)$ for $1 \leq p \leq \infty$ is the space of measurable maps $u: \Omega \rightarrow X$ such that the integral $\int_{\Omega} d\left(u(\omega), x_{0}\right)^{p} d \mu(\omega)$ is finite. This space is a complete metric space with distance satisfying

$$
d(u, v)^{p}=\int_{\Omega} d(u(\omega), v(\omega))^{p} d \mu(\omega)
$$

and if $(X, d)$ is $\operatorname{CAT}(0)$ then so is $L^{2}(\Omega, X)$.

Let $\Gamma$ be the fundamental group of a compact Riemannian manifold $(M, g)$ and let $\rho: \Gamma \rightarrow \operatorname{Isom}(X)$ be a representation of $\Gamma$. The group $\Gamma$ acts by deck transformations on the universal covering $\tilde{M}$ of $M$. We denote by $L_{\rho}^{p}(\tilde{M}, X)$ the space of measurable equivariant maps $u: \tilde{M} \rightarrow X$ such that the restriction of $u$ to a compact fundamental domain $K \subset \tilde{M}$ is in $L^{p}(K, X)$. For two maps $u, v \in L_{\rho}^{p}(\tilde{M}, X)$, the function $x \mapsto d(u(x), v(x))$ is $\Gamma$-invariant and thus can be 
thought as a function on $M$. The distance on $L_{\rho}^{p}(\tilde{M}, X)$ is given by the relation $d(u, v)^{p}=\int_{M} d(u(x), v(x))^{p} d \mu(x)$ where $\mu$ is the measure associated to the Riemannian metric $g$.

For $u \in L_{\rho}^{p}(\tilde{M}, X)$ and $\varepsilon>0$ the $\varepsilon$-approximate energy at $x \in \tilde{M}$ is defined by

$$
e_{\varepsilon}(x)=\int_{S(x, \varepsilon)} \frac{d(u(x), u(y))^{p}}{\varepsilon^{p}} d \sigma(y)
$$

where $S(x, \varepsilon)$ is the $\varepsilon$-sphere around $x$ and $d \sigma$ is the measure induced by $g$ on $S(x, \varepsilon)$, divided by $\varepsilon^{(\operatorname{dim}(M)-1)}$. Now $u$ is said to have finite energy if $e_{\varepsilon}$ converges weakly to a density energy $e$, which is absolutely continuous with respect to $d \mu$ and has finite $L^{1}$-norm, when $\varepsilon$ goes to 0 . In this case, the energy of $u$ is $E(u)=$ $\int_{M} e(x) d \mu(x)$. A minimizer of the energy functional is called a harmonic map.

In [19, Theorem 2.3.1], Korevaar and Schoen proved the existence of an equivariant harmonic map when the target is a CAT(-1) space under the assumption there is no fixed point at infinity. Actually, a Gromov-hyperbolic metric space, for example a CAT(-1) space, is nothing else than a metric space of telescopic dimension, or rank, at most 1 [2, Introduction]. In the unpublished paper [20], the analog in the higher rank (but finite !) case is proved. We include the original argument.

Theorem 3.1 ([20, Theorem 1]). Let $\Gamma$ be the fundamental group of a compact Riemannian manifold $M$ with universal covering $\tilde{M}$ and let $X$ be a complete $\operatorname{CAT}(0)$ space of finite telescopic dimension. If $\Gamma$ acts by isometries on $X$ without fixed point at infinity then there exists a unique equivariant harmonic map $f: \tilde{M} \rightarrow X$. Moreover, this harmonic map is Lipschitz.

Proof. For $L>0$, let $\mathcal{C}_{L}$ be the set of $\Gamma$-equivariant maps from $\tilde{M}$ to $X$ that are $L$-Lipschitz. Thanks to Theorem 2.6.4 in [18], we fix $L>0$ such that $\mathcal{C}_{L}$ is not empty and one can find a minimizing sequence of the energy in $\mathcal{C}_{L}$. We claim that $\mathcal{C}_{L}$ is a closed convex subset of $L_{\rho}^{2}(\tilde{M}, X)$. Let $u, v \in L_{\rho}^{2}(\tilde{M}, X)$ and let $t \mapsto u_{t}$ be the geodesic segment with endpoints $u$ and $v$. If $u, v$ are $L$-Lipschitz, then the convexity of distance function on $X$ [1, Proposition II.2.2] shows that $u_{t}$ is also $L$-Lipschitz for any $t$. Now, the $\mathrm{L}^{2}$-convergence of a sequence with a common Lipschitz bound implies the uniform convergence of this sequence and since a uniform limit of a sequence of $L$-Lipschitz maps is also $L$-Lipschitz, we obtain that $\mathcal{C}_{L}$ is a closed convex subset of $L_{\rho}^{2}(\tilde{M}, X)$.

Let $x_{0} \in \tilde{M}$ and let $X^{\prime}=\left\{x \in X \mid u\left(x_{0}\right)=x, u \in \mathcal{C}_{L}\right\}$. The convexity of $\mathcal{C}_{L}$ implies that $X^{\prime}$ is a convex subset of $X$. We want to show that for any $x \in X^{\prime}$, 
there exists a unique map $u \in \mathcal{C}_{L}$ that minimizes the energy among maps in $\mathcal{C}_{L}$ such that $u\left(x_{0}\right)=x$. Let $u, v \in \mathcal{C}_{L}$ such that $u\left(x_{0}\right)=v\left(x_{0}\right)=x \in X_{0}$ then we have

$$
\int_{M} d(u, v)^{2} \underset{(\mathrm{PI})}{\leq} C \int_{M}\|\nabla d(u, v)\|^{2} \underset{(\mathrm{CI})}{\leq} C\left[\frac{1}{2}(E(u)+E(v))-E(m)\right]
$$

where $C$ is some positive number and $m$ is the midpoint of the segment $[u, v]$. Actually, inequality (PI) is a Poincaré inequality (Lemma 3.2) for the function $d(u, v)$, which is $2 L$-Lipschitz and vanishes at $x_{0}$, and inequality (CI) is [18, inequality (2.6ii)]. inequality (3.1) shows that an energy minimizing sequence $\left(u_{n}\right)$ with $u_{n}\left(x_{0}\right)=x$ for any $n$ is Cauchy. Therefore, an energy minimizing map in $\left\{u \in \mathcal{C}_{l} \mid u\left(x_{0}\right)=x\right\}$ exists and is unique. Let us denote by $f_{x}$ this map.

We define $I(x)$ to be $E\left(f_{x}\right)$. Now, we aim to show that $I: X^{\prime} \rightarrow \mathbb{R}^{+}$is a convex lower semicontinuous function. Assume this is the case, since $I$ is $\Gamma$-invariant and lower semicontinuous, its lower levelsets $X_{r}:=\left\{x \in X^{\prime} \mid I(x) \leq r\right\}$ are $\Gamma$-invariant closed convex subsets of $X$. Proposition 4.4 in [8] implies that the intersection $\cap_{r>\inf I} I_{r}$ is non empty otherwise the center of directions associated to $\left\{I_{r}\right\}_{r>\inf I}$ would be a $\Gamma$-fixed point at infinity. Since $\cap_{r>\inf I} I_{r} \neq \emptyset$, there is an energy minimizing $\Gamma$-equivariant map, which is unique thanks to inequality (3.1).

From the convexity of $E$, it is clear that $I$ is also convex. Let $r>\inf I$ and let $x \in X$ be a limit point of a sequence $\left(x_{n}\right)$ in $X_{r}$. It suffices to show that $f_{n}:=f_{x_{n}}$ is a Cauchy sequence in $\mathcal{C}_{L}$ to obtain that $I_{r}$ is closed. Let $I_{n}=\inf _{X^{\prime} \cap B(x, 1 / n)} I$. We may assume that $x_{n} \in X^{\prime} \cap B(x, 1 / n)$ and $E\left(f_{n}\right) \leq I_{n}+1 / n$. Now, inequality (CI) in (3.1) implies that

$$
\int_{M}\left|\nabla d\left(f_{n}, f_{m}\right)\right|^{2} \underset{n, m \rightarrow \infty}{\longrightarrow} 0
$$

and Lemma 3.2 applied to the function $d\left(f_{n}, f_{m}\right)-d\left(f_{n}\left(x_{0}\right), f_{m}\left(x_{0}\right)\right)$ allows us conclude that $\left(f_{n}\right)$ is a Cauchy sequence.

Lemma 3.2. If $f: M \rightarrow \mathbb{R}$ is a L-Lipschitz function that vanishes at some point $x_{0} \in M$ then there exists $C>0$ which depends only on $M$ and $L$, such that

$$
\int_{M} f^{2} \leq C \int_{M}\|\nabla f\|^{2}
$$

Proof. Let $R$ be the diameter of $M$. By an abuse of notation, we also denote by $f$ the function $f \circ \exp : T_{x_{0}} M \rightarrow \mathbb{R}$. We denote by $\mu$ the pull-back, by the 
exponential map, of the measure associated to the Riemannian metric on $M$ and we denote by $d x$ the Lebesgue measure on $T_{x_{0}} M$. The measure $\mu$ is absolutely continuous with respect to $d x$, thus there is a positive number $C_{1}$ such that the density $v$ of $\mu$ satisfies $v(x)<C_{1}$ for any $x$ in the $R$-ball around the origin in $T_{x_{0}} M$. We have

$$
\int_{M} f^{2} \leq \int_{B(0, R)} f^{2}(x) d \mu(x) \leq C_{1} \int_{B(0, R)} f(x)^{2} d x .
$$

Moreover,

$$
\begin{aligned}
\int_{B(0, R)} f(x)^{2} d x & =\left.\int_{B(0, R)} \int_{0}^{\|x\|} \frac{d}{d u}\right|_{u=t} f(u x /\|x\|)^{2} d t d x \\
& \leq \int_{B(0, R)} \int_{0}^{R} 2 f(t x /\|x\|) \nabla_{\frac{t x}{\|x\|}} f \cdot \frac{x}{\|x\|} d t d x
\end{aligned}
$$

Now, let $n$ be the dimension of $M$ and let $\sigma$ be the Lebesgue measure on $S^{n-1}$. Using polar coordinates, the fact that $\|\nabla f\| \leq L$ and Hölder inequality, we have for some $C_{2}>0$

$$
\begin{aligned}
\int_{M} f^{2} & \leq 2 C_{1} L \int_{0}^{R} \int_{S^{n-1}} \int_{0}^{R} t\left\|\nabla_{t v} f\right\| d t d \sigma(v) r^{n-1} d r \\
& \leq C_{2} \int_{S^{n-1}} \int_{0}^{R}\left\|\nabla_{t v} f\right\|^{n-1} t^{n-1} d t d \sigma(v)=C_{2} \int_{B(0, R)}\left\|\nabla_{x} f\right\|^{n-1} d x .
\end{aligned}
$$

Once again, using Hölder inequality and the fact that the exponential map is finite to one on $B\left(x_{0}, R\right)$, we have for some $C_{3}, C>0$,

$$
\int_{M} f^{2} \leq C_{3} \int_{B\left(x_{0}, R\right)}\left\|\nabla_{x} f\right\|^{2} d x \leq C \int_{M}\|\nabla f\|^{2} .
$$

\section{Smoothness}

It is a standard fact that the most difficult part to obtain smoothness of weak harmonic maps is the first regularity step, which is the continuity of the harmonic map (see for example $[14,8.4]$ ). In our situation, we already know that the harmonic map is Lipschitz and we can easily adapt the argument given in [6], where the target is the infinite dimensional hyperbolic space. 
Proposition 4.1. Let $\Gamma$ be the fundamental group of a compact Riemannian manifold $M$ with universal covering $\tilde{M}$ and let $N$ be a simply connected complete Riemannian manifold of nonpositive sectional curvature. If $\Gamma$ acts by isometries on $N$ and $f: \tilde{M} \rightarrow N$ is a $\Gamma$-equivariant harmonic map in the sense of Korevaar and Schoen then $f$ is a smooth harmonic map.

Proof. We only sketch the proof with the slight modifications to adapt [6, Proposition 7]. We already know that $f$ is Lipschitz. Choose a point $x \in N$. Since $N$ is a simply connected Riemannian manifold of nonpositive curvature, the CartanHadamard Theorem [22, IX.3.8] implies that the exponential map at $x$ is a diffeomorphism from the tangent space $T_{x} N$ to $N$. This gives us a global chart and we can think of $N$ as a Hilbert space $(\mathcal{H},\langle\rangle):,=\left(T_{x} N, h_{x}\right)$ with a non constant Riemannian metric $h$. Moreover, since $N$ has nonpositive sectional curvature, for any $v \in \mathcal{H}$ and any point $y \in N$,

$$
h_{y}(v, v) \geq\langle v, v\rangle,
$$

see [22, Theorem IX.3.6]. In this chart, the covariant derivative can be expressed by

$$
\nabla_{Y} X=D_{Y} X+\Gamma(\exp )(X, Y)
$$

where $\Gamma(\exp )$ is the Christoffel symbol of this chart. Let $B$ be a ball in $\tilde{M}$ of radius less than the injectivity radius of $M$. This way, the projection $\tilde{M} \rightarrow M$ identifies $B$ with a ball in $M$. Consider $f$ as a map from $\tilde{M}$ to $\mathcal{H}$. inequality (4.1) shows that $\left.f\right|_{B}$, which has finite energy for the distance induced by $h$, has finite energy for the one induced by $\langle$,$\rangle , too. Thus, \left.f\right|_{B}$ is in the usual Sobolev space (for vector valued maps) $W^{1,2}(B, \mathcal{H})$. Since $f$ is harmonic, it satisfies the equation

$$
\Delta_{h} f+\sum_{i, j=1}^{\operatorname{dim}(M)} h^{i j} \Gamma(\exp )\left(\frac{\partial f}{\partial x_{i}}, \frac{\partial f}{\partial x_{j}}\right)=0
$$

weakly. An induction on $k$ shows that $\left.f\right|_{B}$ is in $W^{k, p}(B, \mathcal{H})$ for any $k \in \mathbb{N}$ and $p>1$. This shows that $f$ is actually smooth.

\section{A vanishing theorem}

Let $\tilde{M}$ be an irreducible symmetric space of noncompact type that is not the real or complex hyperbolic space and let $\Gamma$ be a uniform torsion free lattice of $\operatorname{Isom}(\tilde{M})$. In order to prove a geometric statement of superrigidity in the cocompact Archimedean case, Mok, Siu and Yeung proved the existence [27] of 
a 4-tensor $Q$ on $\tilde{M}$ that satisfies strong conditions and a Bochner-type formula,

$$
\int_{M}\left\langle\left(Q \circ \sigma_{24}\right) \otimes f^{*} h^{*}, \nabla d f \otimes \nabla d f\right\rangle=1 / 2 \int_{M}\left\langle Q, f^{*} R^{N}\right\rangle .
$$

This formula [27, Theorem 3] holds for an equivariant map $f: \tilde{M} \rightarrow N$ where $N$ is a smooth Riemannian manifold of finite dimension and $Q \circ \sigma_{24}(X, Y, Z, T)=$ $Q(X, T, Z, Y)$. The scalar products are those induced by the Riemannian metrics of $M$ and $N$ on $\left(T^{*} M\right)^{\otimes 4} \otimes\left(f^{-1} T N\right)^{\otimes 2}$ and $\left(T^{*} M\right)^{\otimes 4}$. Actually, the proof of this formula goes through in the case where $N$ has infinite dimension, without modification. The conditions satisfied by $Q$ imply that the right-hand side of equation (5.1) is nonpositive and the harmonicity of $f$ implies that the left-hand side is positive unless $\nabla d f$ vanishes. Thus $f$ is totally geodesic.

Proof of Theorem 1.2. Let $\tilde{M}$ be the symmetric space associated to $G$. Since $\Gamma$ is a torsion free uniform lattice, the quotient space $\Gamma \backslash \tilde{M}$ is a compact manifold. Since $\tilde{M}$ has no fixed point at infinity of $N$, there exists a equivariant harmonic map $f: \tilde{M} \rightarrow N$ by Theorem 3.1. Thanks to Proposition 4.1, we know that $f$ is a smooth equivariant harmonic map.

Assume first that $G$ is simple, that is to say $\tilde{M}$ is irreducible. Now, Mok-SiuYeung above argument implies that $f$ is totally geodesic. Since $\Gamma$ does not fix a point in $N, f(N)$ is not reduced to a point. Now, since $M$ is irreducible, $f$ is an isometry up to rescaling the metric on $M$ (see for example [31]).

Now, if $\tilde{M} \simeq \tilde{M}_{1} \times \cdots \times \tilde{M}_{n}$ with $n \geq 2$ then thanks to a Bochner formula [27, 11], it is proved that the restriction of $f$ to any fiber $x_{1} \times \cdots \times x_{i-1} \times \tilde{M}_{i} \times x_{i+1} \times$ $\cdots \times x_{n}$ is harmonic. The irreducibility of $\Gamma$ allows the authors of [27] to prove that $f$ is actually totally geodesic and thus $f$ factorizes through

$$
\tilde{M} \stackrel{\pi}{\longrightarrow} \prod_{i \in I} \tilde{M}_{i} \stackrel{f^{\prime}}{\longrightarrow} N
$$

where $I$ is a non empty subset of $\{1, \ldots, n\}, \pi$ is the canonical projection and $f^{\prime}$ is an isometry (after renormalization of the metric on each factor $\tilde{M}_{i}$ for $i \in I$ ).

We now explain Remark 1.3. Let $(N, h)$ be a Riemannian manifold with Riemann tensor $R$. Let $X, Y$ be vectors in the complexified tangent space $T_{x} N \otimes \mathbb{C}$ at $x \in N$. We also denote by $R$ and $h$ the $\mathbb{C}$-linear extensions of the Riemann tensor and the metric to the complexification of $T_{x} N$. The complexified sectional 
curvature between $X$ and $Y$ is

$$
\operatorname{Sec}_{\mathbb{C}}(X, Y)=\frac{R(X, Y, \bar{X}, \bar{Y})}{\|X \wedge Y\|_{\mathbb{C}}^{2}}
$$

where \|\|$_{\mathbb{C}}$ is the Hermitian norm on $\wedge^{2}\left(T_{x} N \otimes \mathbb{C}\right)$ induced by the real scalar product

$$
\langle X \wedge Y, Z \wedge T\rangle_{h}=\operatorname{det}\left[\begin{array}{ll}
h(X, Z) & h(X, T) \\
h(Y, Z) & h(Y, T)
\end{array}\right]
$$

on $\wedge^{2} T_{x} N$. The Riemannian manifold $N$ is said to have nonpositive complexified sectional curvature if $\operatorname{Sec}_{\mathbb{C}}(X, Y) \leq 0$ for any $X, Y \in T_{x} N \otimes \mathbb{C}$.

The result of [27], which is the existence of a tensor $Q$ that implies the vanishing of $\nabla d f$ for a harmonic map $f$, is true when $N$ has nonpositive complexified sectional curvature and $G$ is the connected component of the isometry group of the quaternionic hyperbolic space or the Cayley hyperbolic plane. Thus Theorem 1.2 is also true in this case.

Assume that $N$ is a symmetric space and let $C$ be the curvature operator of $N$ as introduced in $[7,3.2]$. We also denote by $C$ its $\mathbb{C}$-linear extension to $T_{x} N \otimes \mathbb{C}$. For $X, Y \in T_{x} N \otimes \mathbb{C}$,

$$
\operatorname{Sec}_{\mathbb{C}}(X, Y)=\frac{\langle C(X \wedge Y), \overline{X \wedge Y}\rangle_{h}}{\|X \wedge Y\|_{\mathbb{C}}^{2}} .
$$

In the case where $N$ is a symmetric space of noncompact type, $C$ is nonpositive and thus, the complexified sectional curvature is nonpositive.

\section{A flat torus theorem for parabolic isometries}

We start with some preliminary results.

Lemma 6.1. Let $X$ be a $\pi$-visible complete $\mathrm{CAT}(0)$ space. If $Y \subseteq X$ is closed and convex then it is also $\pi$-visible.

Proof. Let $\xi, \eta \in \partial Y$ such that $\angle(\xi, \eta)=\pi$. There exists a geodesic $c: \mathbb{R} \rightarrow X$ such that $c(\infty)=\xi$ and $c(-\infty)=\eta$. Let $x$ be the projection of $c(0)$ on $Y$. We define $c_{+}$(respectively $c_{-}$) to be the geodesic ray from $x$ toward $\xi$ (respectively $\eta$ ). By definition of the boundary, $d\left(c(t), c_{+}(t)\right)$ and $d\left(c(-t), c_{-}(t)\right)$ are bounded for 
$t \geq 0$. The real function $t \mapsto d(c(t), Y)$ is convex bounded and thus constant equal to some $d_{0} \geq 0$. Now let $c^{\prime}(t)$ be the projection of $c(t)$ on $Y$ for $t \in \mathbb{R}$. For $s, t \in \mathbb{R}$ and $x \in[c(t), c(s)], d\left(x,\left[c^{\prime}(t), c^{\prime}(s)\right]\right)=d_{0}$. By the same argument as above, for all $x \in\left[c^{\prime}(t), c^{\prime}(s)\right], d(x,[c(t), c(s)])=d_{0}$ and we are in position to apply the Sandwich Lemma [1, II.2.12.(2)], which shows that the convex hull of $c(t), c(s), c^{\prime}(s), c^{\prime}(t)$ is a Euclidean rectangle. In particular, $c^{\prime}: \mathbb{R} \rightarrow Y$ is a geodesic with $c^{\prime}(\infty)=\xi$ and $c^{\prime}(-\infty)=\eta$.

We recall that a ballistic isometry $\gamma$ of a complete CAT(0) space $X$ has two canonical fixed points at infinity, which we denote by $\omega_{\gamma}$ and $\omega_{\gamma^{-1}}$. They are limit points at infinity of $\gamma^{n} x$ and $\gamma^{-n} x$ for any $x \in X$ (see [3,3.C], for example).

Proposition 6.2. Let $X$ be a $\pi$-visible complete $\mathrm{CAT}(0)$ space and let $\gamma$ be a ballistic isometry of $X$. Then there exists a closed convex subspace $Y \subseteq X$ that splits as $Z \times \mathbb{R}$. Moreover, $Z_{\operatorname{Isom}(X)}(\gamma)$ preserves $Y$ and acts diagonally. In particular, $\left.\gamma\right|_{Y}$ acts as a translation of length $|\gamma|$ along the factor $\mathbb{R}$.

Proof. Let $Y$ be the union of geodesics with endpoints $\omega_{\gamma}$ and $\omega_{\gamma^{-1}}$. Since $X$ is $\pi$-visible, $Y$ is nonempty and $\gamma$-invariant. Moreover, $Y$ is a closed subspace of $X$. Let $x \in X$ be a limit point of a sequence $\left(x_{n}\right)$ of points in $Y$. Let $c_{n}$ be the geodesic such that $c_{n}(0)=x_{n}, c_{n}(-\infty)=\omega_{\gamma^{-1}}$ and $c_{n}(\infty)=\omega_{\gamma}$. Thanks to [1, Proposition II.9.22], $c_{n}$ converges to a geodesic $c$ such that $c(0)=x, c(-\infty)=\omega_{\gamma^{-1}}$ and $c(\infty)=\omega_{\gamma}$.

Since $Y$ is closed, convex and $\gamma$-invariant, $|\gamma|_{Y}|=| \gamma \mid$. The subspace $Y$ decomposes as a product $Y \simeq Z \times \mathbb{R}$ (see [1, Theorem II.2.14]) and $\gamma$ preserves this decomposition. Thus $\left.\gamma\right|_{Y}$ can be written $\gamma_{0} \times \gamma_{1}$. A simple computation shows that $|\gamma|^{2}=\left|\gamma_{0}\right|^{2}+\left|\gamma_{1}\right|^{2}$. Assume for contradiction that $\left|\gamma_{0}\right|>0$ then there exists $\omega_{\gamma_{0}} \in \partial Z$ such that $\gamma_{0}^{n} x_{0} \rightarrow \omega_{\gamma_{0}}$ for any $x_{0} \in Z$. Thus, for any $x \in Y$, $\gamma^{n} x \rightarrow\left(\arccos \left(\left|\gamma_{0}\right| /\left|\gamma_{1}\right|\right), \omega_{\gamma_{0}}, \omega_{\gamma}\right)$ in the spherical join $\partial Z * \partial \mathbb{R}=\partial Y$.

Lemma 6.3. Let $A$ be an abelian group acting by isometries on a $\mathrm{CAT}(0)$ space $X$. The set $N$ of neutral elements in $A$ is a subgroup of $A$. Moreover, if $A \simeq \mathbb{R}^{n}$ and the action is continuous then it is a linear subspace of $A$.

Proof. We recall that $|a|=\lim _{n \rightarrow \infty} \frac{d(\operatorname{nax}, x)}{n}$ for any $x \in X$. Thus $|a+b|=\lim _{n} \frac{d(n(a+b) x, x)}{n} \leq \lim _{n} \frac{d(n a(n b x), n a x)+d(\text { nax }, x)}{n} \leq|a|+|b|$. Moreover, $|a|=|-a|$ and $|n a|=n|a|$ for any $n \in \mathbb{N}$. Thus, if $A \simeq \mathbb{R}^{n}$, by continuity $|\lambda a|=|\lambda||a|$ for any $\lambda \in \mathbb{R}$ and $a \in A$. 
We are ready to prove Theorem 1.4.

Proof of Theorem 1.4. We prove this theorem by induction on $n$. For $n=1$, this is Proposition 6.2. Now suppose $n \geq 2$ and choose a primitive element $a \in$ $A$ and apply Proposition 6.2. We obtain an $Z_{\operatorname{Isom}(X)}(a)$-invariant closed convex subspace $Y_{1} \simeq Z_{1} \times \mathbb{R}$ and $a$ acts as a translation of length $|a|$ on the $\mathbb{R}$-factor. The group $Z_{\text {Isom }(X)}(a)$ acts also diagonally on $Y_{1}$. Let $N$ be the subset of $A$ formed by elements $b=\left(b_{1}, b_{2}\right)$ of $A$ such that $\left|b_{1}\right|=0$. Lemma 6.3 shows $N$ is a subgroup of $A$. The subgroup $N$ acts properly on $\mathbb{R}$ and thus is cyclic. It contains $a$ and since $a$ is primitive in $A, N=a \mathbb{Z}$. Now let $B$ be a free abelian group of rank $n-1$ such that $A=N \oplus B$. Observe that $B$ acts by ballistic isometries on $Z_{1}$. We can now apply an induction for $B \curvearrowright Z_{1}$ and we obtain $Y_{2} \simeq Z \times \mathbb{R}^{n-1} \subseteq$ $Z_{1}$. By induction $Y_{2}$ is $Z_{\operatorname{Isom}\left(Z_{1}\right)}(B)$-invariant and $Z_{\operatorname{Isom}\left(Z_{1}\right)}(B)$ preserves this decomposition. Moreover, for any $\gamma \in Z_{\operatorname{Isom}(X)}(A), \gamma_{1} \in Z_{\operatorname{Isom}\left(Z_{1}\right)}(B)$ and thus $\gamma_{1}$ preserves $Y_{2}$ and acts diagonally on it. In particular, $a_{1}$ (which is neutral) has a trivial part on $\mathbb{R}^{n-1}$. Now if we set $Y=Y_{2} \times \mathbb{R} \simeq Z \times \mathbb{R}^{n} \subseteq X, Y$ has the desired properties.

Proof of Corollary 1.5. Thanks to [30, Corollary 2.9], $\Gamma$ contains an Abelian free group of rank $r$. Since this Abelian free group acts also by ballistic isometries, it suffices to apply Theorem 1.4 to find a Euclidean subspace of $X_{p}(\mathbb{K})$ of dimension $r$ and thus $p \geq r$.

\section{References}

[1] M. R. Bridson and A. Haefliger, Metric spaces of non-positive curvature. Grundlehren der Mathematischen Wissenschaften, 319. Springer, Berlin etc., 1999. Zbl 0988.53001 MR 1744486

[2] P.-E. Caprace and A. Lytchak, At infinity of finite-dimensional CAT(0) spaces. Math. Ann. 346 (2010), no. 1, 1-21. Zbl 1184.53038 MR 2558883

[3] P.-E. Caprace and N. Monod, Isometry groups of non-positively curved spaces: structure theory. J. Topol. 2 (2009), no. 4, 661-700. Zbl 1209.53060 MR 2574740

[4] K. Corlette, Flat G-bundles with canonical metrics. J. Differential Geom. 28 (1998), 361-382. Zbl 0676.58007 MR 0965220

[5] K. Corlette, Archimedean superrigidity and hyperbolic geometry. Ann. of Math. (2) 135 (1992), no. 1, 165-182. Zbl 0768.53025 MR 1147961

[6] Th. Delzant and P. Py, Kähler groups, real hyperbolic spaces and the cremona group. Compos. Math. 148 (2012), no. 1, 153-184. Zbl 06007113 MR 2881312 
[7] B. Duchesne, Infinite dimensional Riemannian symmetric spaces with fixed-sign curvature operator. Preprint 2012. arXiv:1204.6012 [math.DG]

[8] B. Duchesne, Infinite-dimensional nonpositively curved symmetric spaces of finite rank. Int. Math. Res. Not. IMRN 2013 (2013), no. 7, 1578-1627. MR 3044451

[9] T. Gelander, A. Karlsson, and G. A. Margulis, Superrigidity, generalized harmonic maps and uniformly convex spaces. Geom. Funct. Anal. 17 (2008), no. 5, 1524-1550. Zbl 1156.22005 MR 2377496

[10] M. Gromov and P. Pansu, Rigidity of lattices: an introduction. In P. De Bartolomeis and F. Tricerri (eds.), Geometric topology: recent developments. Lecture Notes in Mathematics, 1504. Springer, Berlin etc., 1991, 39-137. Zbl 0786.22015 Zbl 0746.00065 (collection) MR 1168043 MR 1168041 (collection)

[11] M. Gromov, Asymptotic invariants of infinite groups. In G. A. Niblo and M. A. Roller (eds.), Geometric group theory. Vol. 2. London Mathematical Society Lecture Note Series, 182. Cambridge University Press, Cambridge, 1993, 1-295. Zbl 0841.20039 MR 1253544 MR 1253543 (collection)

[12] M. Gromov and R. Schoen, Harmonic maps into singular spaces and $p$-adic superrigidity for lattices in groups of rank one. Inst. Hautes Études Sci. Publ. Math. 76 (1992), 165-246. Zbl 0896.58024 MR 1215595

[13] J. Jost, Nonpositive curvature: geometric and analytic aspects. Lectures in Mathematics ETH Zürich. Birkhäuser, Basel, 1997. Zbl 0896.53002 MR 1451625

[14] J. Jost, Riemannian geometry and geometric analysis. $6^{\text {th }}$ ed., Universitext. Springer, Berlin etc., 2011. Zbl 1227.53001 MR 2829653

[15] J. Jost and Sh.-T. Yau, Harmonic maps and superrigidity. In R. Greene and Sh.-T. Yau (eds.), Differential geometry: partial differential equations on manifolds. Proceedings of Symposia in Pure Mathematics, 54, Part 1. American Mathematical Society, Providence, R.I., 1993, 245-280. Zbl 0806.58012 Zbl 0773.00022 (collection) MR 1216587 MR 1216572 (collection)

[16] J. Jost and Sh.-T. Yau, Harmonic maps and rigidity theorems for spaces of nonpositive curvature. Comm. Anal. Geom. 7 (1999), no. 4, 681-694. Zbl 0933.53030 MR 1714940

[17] W. P. A. Klingenberg, Riemannian geometry. $2^{\text {nd }}$ ed., de Gruyter Studies in Mathematics, 1. Walter de Gruyter \& Co., Berlin, 1995. Zbl 0911.53022 MR 1330918

[18] N. J. Korevaar and R. M. Schoen, Sobolev spaces and harmonic maps for metric space targets. Comm. Anal. Geom. 1 (1993), no. 3-4, 561-659. Zbl 0862.58004 MR 1266480

[19] N. J. Korevaar and R. M. Schoen, Global existence theorems for harmonic maps to non-locally compact spaces. Comm. Anal. Geom. 5 (1997), no. 2, 333-387. Zbl 0908.58007 MR 1483983

[20] N. J. Korevaar and R. M. Schoen, Global existence theorems for harmonic maps: finite rank spaces and an approach to rigidity for smooth actions. Unpublished, 1999. 
[21] F. Labourie, Existence d'applications harmoniques tordues à valeurs dans les variétés à courbure négative. Proc. Amer. Math. Soc. 111 (1991), no. 3, 877-882. Zbl 0783.58016 MR 1049845

[22] S. Lang, Fundamentals of differential geometry. Graduate Texts in Mathematics, 191. Springer, New York, 1999. Zbl 0932.53001 MR 1666820

[23] U. Lang and V. Schroeder, Jung's theorem for Alexandrov spaces of curvature bounded above. Ann. Global Anal. Geom. 15 (1997), no. 3, 263-275. Zbl 0974.53028 MR 1456512

[24] G. A. Margulis, Discrete subgroups of semisimple Lie groups. Ergebnisse der Mathematik und ihrer Grenzgebiete (3), 17. Springer, Berlin etc., 1991. Zbl 0732.22008 MR 1090825

[25] N. Monod, Superrigidity for irreducible lattices and geometric splitting. J. Amer. Math. Soc. 19 (2006), no. 4, 781-814. Zbl 1105.22006 MR 2219304

[26] N. Monod and P. Py, An exotic deformation of the hyperbolic space. Amer. J. Math. 136 (2014), no. 5, 1249-1299. Zbl 06365640 MR 3263898

[27] N. Mok, Y. T. Siu, and S.-K. Yeung, Geometric superrigidity. Invent. Math. 113 (1993), no. 1, 57-83. Zbl 0808.53043 MR 1223224

[28] S. Nishikawa, Variational problems in geometry. Translated from the 1998 Japanese original by Kinetsu Abe. Translations of Mathematical Monographs, 205. Iwanami Series in Modern Mathematics. American Mathematical Society, Providence, RI, 2002. Zbl 1010.53001 MR 1896863

[29] P. Pansu, Superrigidité géométique et applications harmoniques. In L. Bessières, A. Parreau, and B. Rémy (eds.), Géométries à courbure négative ou nulle, groupes discrets et rigidités. Séminaires et Congrès, 18. Société Mathématique de France, Paris, 2009. 373-420. Zbl 1201.53051 Zbl 1186.53001 (collection) MR 2655318 MR 2664216 (collection)

[30] G. Prasad and M. S. Raghunathan, Cartan subgroups and lattices in semi-simple groups. Ann. of Math. (2) 96 (1972), 296-317. Zbl 0245.22013 MR 0302822

[31] J. Vilms, Totally geodesic maps. J. Differential Geometry 4 (1970), 73-79. Zbl 0194.52901 MR 0262984

Received July 2, 2012

Bruno Duchesne, Einstein Institute of Mathematics, J. Safra Campus, Givat Ram, The Hebrew University of Jerusalem, Jerusalem, 91904, Israel,

Current address: Institut Elie Cartan, Université de Lorraine, 54506

Vandoeuvre-lès-Nancy, France

e-mail: bruno.duchesne@ens-lyon.org 